\title{
Mit Fadenlift und Filler Konturen definieren
}

\begin{abstract}
Die Kombination ästhetischer Behandlungskonzepte gewinnt an Bedeutung, so die einhellige Meinung der Experten beim „Expert-Day“. Sie berichteten unter anderem über ihre Erfahrungen mit resorbierbaren Fadenlift-Systemen und Fillern mit wählbarer Haltbarkeit auf Milchsäure-Basis.

Der plastische Chirurg Dr. Roberto Pizzamiglio, Krankenhaus Quirón Marbella, Spanien, demonstrierte, wie durch das Fadenlift mit Silhouette Soft ${ }^{\oplus}$ Gesichtskonturen geformt werden können. Nicht nur die Haut werde gestrafft, sondern auch ein Volumenverlust im Wangenbereich bzw. Mittelgesicht kompensiert. Es sei möglich, die Unterkieferpartie zu formen und die sogenannten Hängebäckchen, die im Alter durch Rückbildung des Unterkieferknochens entstünden, auszugleichen. Die Methode eigne sich auch, um die Brauenlinie sanft anzuheben oder
\end{abstract}

den Halsbereich zu straffen. Hauptbestandteil der resorbierbaren Fäden und der Kegel ist Polymilchsäure (PLA), die innerhalb von zwei Jahren abgebaut werde. Die kleinen Kegel sind auf den Faden von der Mitte aus in entgegengesetzte Richtungen aufgefädelt (bidirektional). Durch die Verankerung über die Kegel wird der Faden im Gewebe fixiert. Das Einbringen der Fäden habe einen doppelten Effekt: sofortige Anhebung, da das Gewebe komprimiert und die Haut gestrafft werde und zusätzlich stimuliere die Resorption des Fadenmaterials die Kollagenproduktion.

Die Fäden sollten stets ins subkutane Fettgewebe (5 mm tief) eingebracht werden. Wichtig sei es auch, den Faden nicht zu stark zu straffen. Als Risikoregionen, auf die es bei der Injektion zu achten gelte, nannte Pizzamiglio die Bereiche der Rami temporales, R. buccales und R. mandibularis des Nervus facialis. Insgesamt sei der Effekt dezenter als bei einem Facelift, so Pizzamiglio. Zudem sei es eine Behandlung und kein chirurgischer Eingriff, betonte er: Die Einstichstellen heilten nach wenigen Tagen ab und eine Lokalanästhesie (Lidocain $1 \%$ plus Adrenalin 1:200.000) reiche aus.

Für eine komplexe dreidimensionale ästhetische Gestaltung könne die Methode auch mit den Kollagenstimulatoren des Ellansé $^{\mathrm{rm}}$-Sortiments kombiniert werden, riet Pizzamiglio. Die Filler bestehen zu $70 \%$ aus wässriger Carboxymethylcellulose (CMC) und zu $30 \%$ aus synthetischen Polycaprolacton-(PCL-)Mikropartikeln. Ihre Haltbarkeit beträgt je Länge der Polymerkette ein bis vier Jahre. CMC sorgt für einen sofortigen Fülleffekt, der etwa drei Monate vorhalte, während PCL die körpereigene Kollagensynthese stimuliere. Pizzamiglio empfahl, zunächst den Filler anzuwenden und zwei bis drei Monate später die Fäden einzusetzen. Susanne Pickl

Symposium „Sinclair Expert Day", Frankfurt am Main, 4.6.2016; Veranstalter: Sinclair Pharma

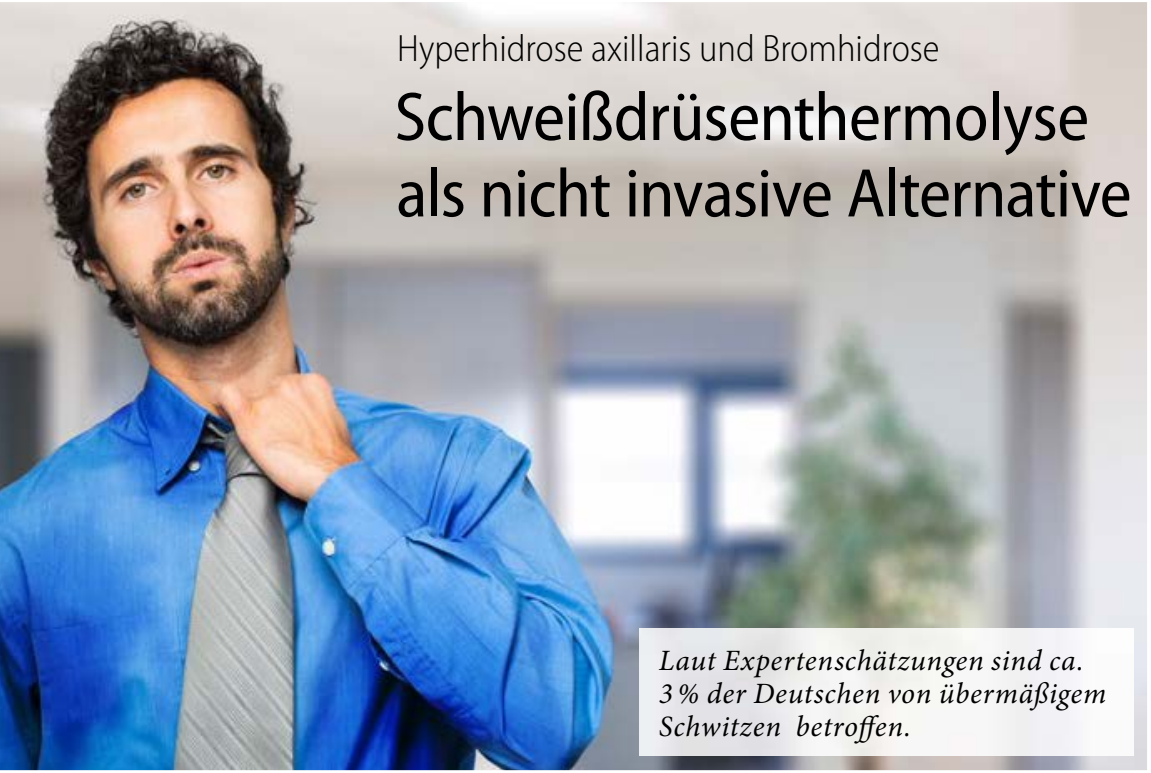

Schwitzen ist ein überlebenswichtiger Mechanismus, der an der Regulierung der Körpertemperatur wesentlich beteiligt ist. Laut Expertenschätzungen sind ca. $3 \%$ der Deutschen von übermäßigem Schwitzen betroffen. Hyperhidrose entsteht in der Regel durch eine Überstimulation ekkriner Schweißdrüsen (primäre Hyperhidrosis). Eine Überfunktion apokriner Schweißdrüsen (Bromhidrose) kann zusätzlich noch zu starkem Körpergeruch führen.

Die Schweißdrüsenthermolyse durch elektromagnetische Energie (miraDry ${ }^{\circledR}$ ) ist eine Alternative $\mathrm{zu}$ herkömmlichen Behandlungsmethoden der Hyperhidrosis axillaris. Das nicht invasive, klinisch erprobte Verfahren basiert auf kontrollierter Applikation von Mikrowellen. Durch die Lokalanästhesie ist der Pro- zess schmerzfrei. Die Behandlung wird unter Tumeszens-Anästhesie durchgeführt. Nach 10 Minuten Einwirkzeit beginnt die Behandlung mit dem Handstück.

Durch das Thermolyse-Verfahren wird die obere Subkutis mit Mikrowellen der Wellenlänge 5,8 MHz bestrahlt. Durch Interferenzen bildet sich eine fokale Energiezone. Unter Einwirkung der Temperatur von ca. $60^{\circ} \mathrm{C}$ setzt die Zellthermolyse ein. Das hydrokeramische Kühlsystem begrenzt die Wärmezone auf den Bereich der Schweißdrüsen.

Mögliche Nebenwirkungen, wie etwa vorübergehende Taubheit im Behandlungsareal, sind nur kurzzeitig, sodass keine Ausfallzeiten entstehen. Klinische Langzeitstudien bestätigen, dass bereits nach einer Behandlung das Schwitzen im Achselbereich um ca. $82 \%$ reduziert wird. Das Verfahren ist derzeit die einzige Thermolyse-Methode, die von der Food and Drug Administration (FDA) zugelassen ist, ein CE-Zeichen besitzt und vom Deutschen Hyperhidrosezentrum sowie der International Hyperhidrosis Society empfohlen wird.

red

Nach Informationen von Miramar Labs 